\title{
Optimization of a Direct Analysis in Real Time/ Time-of-Flight Mass Spectrometry Method for Rapid Serum Metabolomic Fingerprinting
}

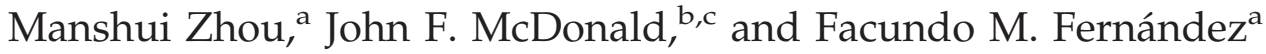 \\ a School of Chemistry and Biochemistry, Georgia Institute of Technology, Atlanta, Georgia, USA \\ ${ }^{\mathrm{b}}$ School of Biology, Georgia Institute of Technology, Atlanta, Georgia, USA \\ c Ovarian Cancer Institute, Atlanta, Georgia, USA
}

\begin{abstract}
Metabolomic fingerprinting of bodily fluids can reveal the underlying causes of metabolic disorders associated with many diseases, and has thus been recognized as a potential tool for disease diagnosis and prognosis following therapy. Here we report a rapid approach in which direct analysis in real time (DART) coupled with time-of-flight (TOF) mass spectrometry (MS) and hybrid quadrupole TOF (Q-TOF) MS is used as a means for metabolomic fingerprinting of human serum. In this approach, serum samples are first treated to precipitate proteins, and the volatility of the remaining metabolites increased by derivatization, followed by DART MS analysis. Maximum DART MS performance was obtained by optimizing instrumental parameters such as ionizing gas temperature and flow rate for the analysis of identical aliquots of a healthy human serum samples. These variables were observed to have a significant effect on the overall mass range of the metabolites detected as well as the signal-to-noise ratios in DART mass spectra. Each DART run requires only 1.2 min, during which more than 1500 different spectral features are observed in a time-dependent fashion. A repeatability of $4.1 \%$ to $4.5 \%$ was obtained for the total ion signal using a manual sampling arm. With the appealing features of high-throughput, lack of memory effects, and simplicity, DART MS has shown potential to become an invaluable tool for metabolomic fingerprinting. (J Am Soc Mass Spectrom 2010, 21, 68-75) @ 2010 American Society for Mass Spectrometry
\end{abstract}

$\mathrm{M}$ etabolomic fingerprinting, an unbiased, global screening approach to classify samples based on metabolite patterns or "fingerprints", has been performed in a wide variety of biological sample types such as urine, plasma, serum, and tissues [1]. Nuclear magnetic resonance (NMR) and mass spectrometry are two widely used analytical platforms applied to metabolomic fingerprinting. NMR has the advantage of requiring little sample preparation, and of producing datasets that are more easily mined [2-4], but cost and sensitivity are two of its major limitations. Gas chromatography-mass spectrometry (GC-MS) and liquid chromatography-mass spectrometry (LC-MS) are two additional techniques commonly used in metabolomic workflows [1, 5-9]. Despite being extremely comprehensive, GC-MS and LC-MS suffer from low analysis throughput and memory effects of the chromatographic supports, particularly when investigating metabolites in biological matrices such as serum. To overcome the above described limitations, enabling technologies for the effective analysis of metabolomes are still rapidly evolving.

Direct analysis in real time (DART) is a plasma-based ion generation technique that operates under ambient

Address reprint requests to Dr. F. M. Fernández, School of Chemistry and Biochemistry, Georgia Institute of Technology, 901 Atlantic Dr. NW, Atlanta, GA 30332-0400, USA. E-mail: facundo.fernandez@chemistry.gatech.edu conditions. It belongs to a wider family of ambient plasma ionization techniques that includes direct atmospheric pressure photo-ionization (DAPPI) [10], direct analysis in real time (DART) [11], flowing atmospheric pressure afterglow (FAPA) [12], plasma-assisted desorption ionization (PADI) [13], desorption atmospheric pressure photoionization (DAPCI) [14], low-temperature plasma (LTP) ionization [15], and dielectric barrier discharge ionization (DBDI) [16]. DART enables ionization in a noncontact fashion with minimal memory effects. Samples are handled either manually or with the assistance of autosamplers, and mainly high purity compressed gases ( $\mathrm{He}$ or $\mathrm{N}_{2}$ ) are required as consumables resulting in low cost per sample [11]. DART operates by first creating a glow discharge within a flowing stream of gas $\left(\mathrm{N}_{2}\right.$ or $\left.\mathrm{He}\right)$ to form metastables that interact with water in the atmosphere to produce protonated water clusters. These clusters react by proton transfer with analytes desorbed by the heated gas stream. In most cases, immediate ionization can be achieved with no sample preparation. DART has been successfully applied to the analysis of pharmaceuticals [17, 18], counterfeit drugs [19], bacterial fatty acid methyl esters [20], flavors and fragrances [21], and several other interesting applications [22, 23].

In this work, we report a method for the rapid metabolomic fingerprinting of human serum via 
DART-TOF and DART-Q-TOF MS. The optimization of various experimental parameters is discussed, and the identification of metabolites in serum is demonstrated using accurate mass measurements and spiking experiments. To the best of our knowledge, this is the first report on the coupling of a DART ion source to a hybrid Q-TOF mass spectrometer, and on the use of DART for serum metabolomics.

\section{Experimental}

\section{Samples and Reagents}

N-trimethylsilyl-N-methyltrifluoroacetamide (MSTFA) and trimethylchlorosilane (TMCS) were obtained from Alfa Aesar (Ward Hill, MA, USA), anhydrous pyridine, acetonitrile $(\mathrm{ACN})$, acetone, and isopropanol were from EMD Chemicals (Gibbstown, NJ, USA), polyethylene glycol standard 400 and 600 (PEG 400, 600) were from Fluka Chemical Corp. (Milwaukee, WI, USA), healthy human serum (S7023-50 mL), creatinine, urea, cysteamine, triethylamine, L-proline, L-alanine, L-arginine, Lserine, and L-lysine were purchased from Sigma-Aldrich Corp. (St. Louis, MO, USA), pyroglutamic acid was from AnaSpec (San Jose, CA, USA), 3-indolebutyric acid was from TCI America (Wellesley Hills, MA, USA), and helium (99.999\% purity) was purchased from Airgas, Inc. (Atlanta, GA, USA).

\section{Mass Spectrometry}

Serum metabolomic analysis was performed in positive ion mode via a DART ion source (IonSense, Saugus, MA, USA) coupled to (1) an orthogonal time-of-flight (TOF) mass spectrometer (JEOL AccuTOF; Tokyo, Japan) and (2) a Q-TOF mass spectrometer (Bruker micrOTOF-Q I; Bremen, Germany). The Q-TOF mass spectrometer interface was modified before coupling to DART by adding to its front end a custom made VAPUR gas-ion separator tube (IonSense, Saugus, MA, USA). This VAPUR interface was itself connected to an Edwards E2M2 roughing pump (Edwards, Westminster, MD, USA) to remove the excess discharge gas flowing into the mass spectrometer inlet. The AccuTOF TOF mass spectrometer was interfaced to the DART ion source directly through a small orifice $(\sim 375 \mu \mathrm{m}$ diameter) interface. In both cases, derivatized serum samples were placed within the ionization region using a homebuilt sampling arm which secured Dip-it tips (IonSense, Saugus, MA, USA) at a fixed $3 \mathrm{~mm}$ distance from the ion source gas exit. Before DART MS analysis, $0.5 \mu \mathrm{L}$ of derivatized serum solution was pipette-deposited onto the glass end of the Dip-tip coupled to the sampling arm, a $1.2 \mathrm{~min}$ data acquisition run started, and the sample allowed to air dry for $0.65 \mathrm{~min}$. The sampling arm was then rapidly switched so that the dried sample was exposed to the ionizing zone of the DART ion source. After $0.9 \mathrm{~min}$ in the acquisition run $(0.25 \mathrm{~min}$ sampling time), the sample was removed, and a new
Dip-it placed on the sample holder, while the remaining 0.3 min of the run was completed.

DART ion source helium flow rates of $3.0 \mathrm{~L} \mathrm{~min}^{-1}$ and $0.55 \mathrm{~L} \mathrm{~min}^{-1}$ heated to $200{ }^{\circ} \mathrm{C}$ were chosen for TOF and Q-TOF experiments, respectively. The glass tip-end was positioned $1.5 \mathrm{~mm}$ below the mass spectrometer inlet for TOF experiments and at the center of the ceramic tube inlet for Q-TOF experiments. A discharge needle voltage of $+3600 \mathrm{~V}$, perforated electrode voltage of $+150 \mathrm{~V}$, and grid electrode voltages of $+250 \mathrm{~V}$ (TOF), and $+50 \mathrm{~V}(\mathrm{Q}-\mathrm{TOF})$ were chosen, respectively. Accurate mass spectra were acquired in the $m / z$ 60-1000 range with a spectral recording interval of $1.0 \mathrm{~s}$. For TOF experiments, the RF ion guide peak voltage was set to $1200 \mathrm{~V}$ to enable ion transmission of a wide range of species present in serum. The remaining settings for the TOF mass spectrometer were as follows: ring lens, +8 $\mathrm{V}$, orifice $1,+40 \mathrm{~V}$, orifice $2,+6 \mathrm{~V}$, orifice 1 temperature, $80^{\circ} \mathrm{C}$, and detector voltage, $-2800 \mathrm{~V}$. TOF mass drift compensation was performed after analysis of each sample using a $0.20 \mathrm{mM}$ PEG 600 standard in methanol. The measured resolving power of the TOF mass spectrometer was 6000 at FWHM, with observed mass accuracies in the range 2-20 ppm, depending on the signal-to-noise ratio $(\mathrm{S} / \mathrm{N})$ of the particular peak under investigation.

The settings for the Q-TOF mass spectrometer were as follows: capillary voltage, $-1500 \mathrm{~V}$, end plate offset, $-500 \mathrm{~V}$, drying gas flow, $2.0 \mathrm{~L} \mathrm{~min}^{-1}$, drying gas temperature, $150{ }^{\circ} \mathrm{C}$. The minimum measured resolving power of the Q-TOF mass spectrometer was 14,500 at FWHM for $m / z 415.25$, with observed mass accuracies in the range $0.6-2.0 \mathrm{ppm}$. The instrument was mass calibrated before performing experiments using a custom mixture of trimethylamine, PEG 400, and 600 in methanol (1:2:2:1200 vol/vol), and all mass spectra were centroided in real time.

Metabolites were tentatively identified by matching accurate masses against a custom built database containing 2924 entries corresponding to unique endogenous human metabolites found in the Human Metabolome Database (HMDB, www.hmdb.ca). Each entry was manually expanded to take into account the mono-, di-, and/or tri-trimethylsilane (TMS) derivatives. Entries for families of compounds not reacting with the MSTFA/TMCS reagent mixture were not expanded. Matching of database accurate mass records to experimental data were performed using the SearchFromList application included in the Mass Spec Tools suite of programs (ChemSW, Fairfield, CA, USA) using a tolerance of $5 \mu \mathrm{m}$ and a relative intensity cutoff of $5 \%$.

\section{Sample Preparation}

Upon removal from a $-80{ }^{\circ} \mathrm{C}$ freezer, serum samples were immediately thawed on ice. Two-hundred $\mu \mathrm{L}$ serum aliquots were pipetted and mixed with $1 \mathrm{~mL}$ of freshly-prepared, chilled $\left(-8{ }^{\circ} \mathrm{C}\right)$ and degassed 2:1 (vol/vol) acetone:isopropanol mixture. The mixture 
was vortexed and placed in a second freezer at $-18^{\circ} \mathrm{C}$ overnight to precipitate proteins, followed by centrifugation at $13,000 \mathrm{~g}$ for $5 \mathrm{~min}$. The supernatant was transferred to a clean centrifuge tube, and the solvent was evaporated in a speed vacuum concentrator to complete dryness for $24 \mathrm{~h}$. The solid residue was then re-dissolved in $25 \mu \mathrm{L}$ anhydrous pyridine, and shaken for $1 \mathrm{~h}$ at room temperature for complete dissolution. Fifty $\mu \mathrm{L}$ of MSTFA containing $0.1 \%$ TMCS were added to the sample in a $\mathrm{N}_{2}$-purged glove box. The mixture was incubated at $50{ }^{\circ} \mathrm{C}$ in an inert $\mathrm{N}_{2}$ atmosphere for 30 min, resulting in derivatization of amide, amine, and hydroxyl groups. The supernatant of this derivatized mixture was subject to DART mass spectrometric analysis.

\section{Results and Discussion}

\section{General Spectral Appearance}

DART-TOF mass spectra for nonderivatized human serum following protein precipitation and for an identical sample derivatized with MSTFA/TMCS are shown in Figure 1. Only about 100 total signals were obtained from nonderivatized serum (Figure 1a), while more than 1500 detectable signals were observed in derivatized serum (Figure 1b). Underivatized serum presented signals mainly in the $\mathrm{m} / \mathrm{z} 100-300$ mass range, whereas for derivatized serum signals up to $\mathrm{m} / \mathrm{z} 800$ were detected due to the enhanced volatility of the TMS metabolite derivatives. Increased volatility facilitates thermal desorption before chemical ionization within the region between the DART ion source exit and the mass spectrometer inlet. Overall signal intensity was increased on average by a factor of 5 following derivatization.

Peaks with relative abundance higher than $5 \%$ of the base peak in Figure 1b were identified based on an analysis of the number of TMS groups incorporated upon derivatization and accurate mass matching of the corresponding species against the custom built metabolite database. HMDB entries matched following this procedure are listed in Supplemental Material Table S-1, which can be found in the electronic version of this article. Twenty-five unknowns were identified. Confirmation of the identity of 10 of these metabolites was performed by spiking the serum sample with a mixture
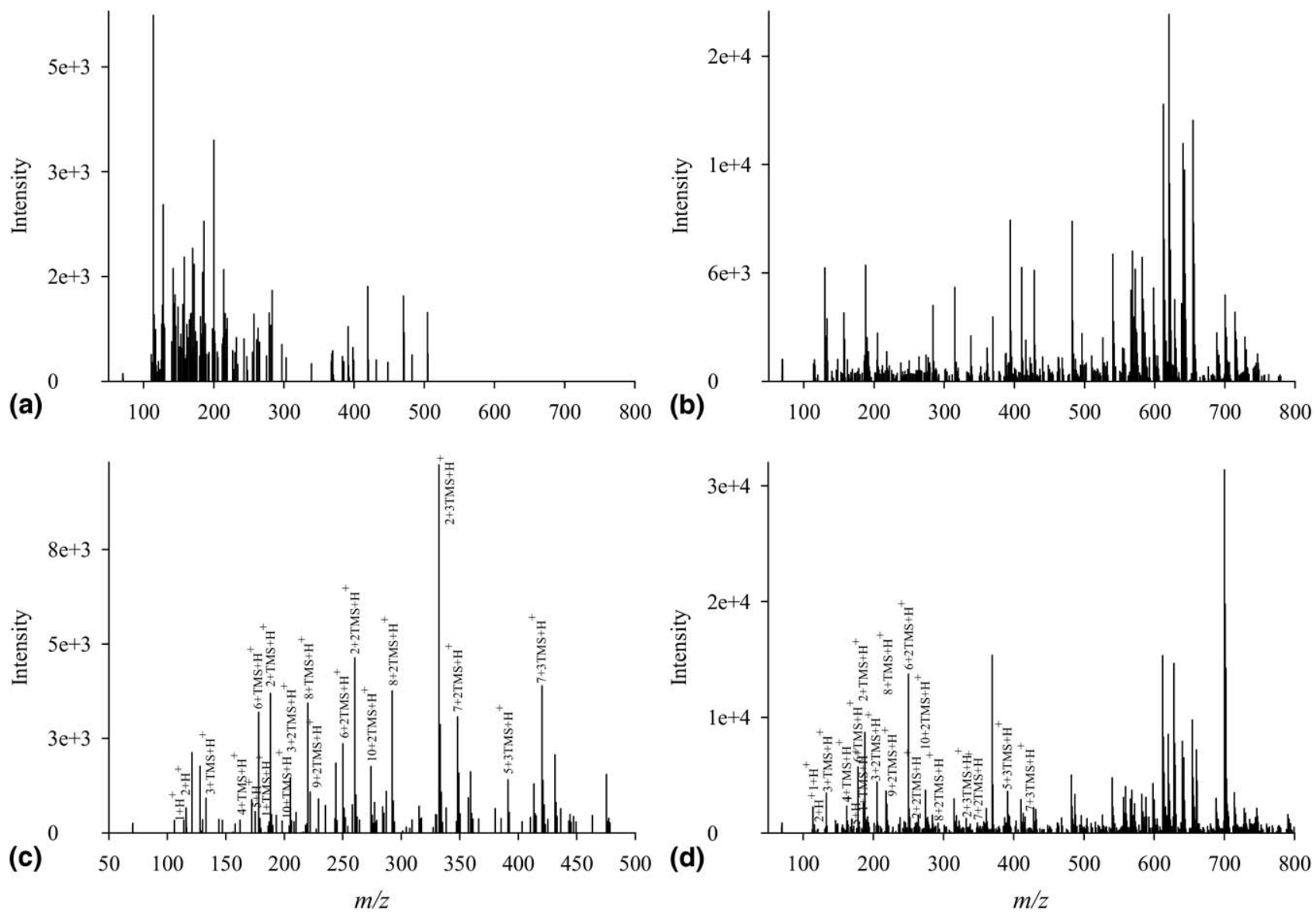

Figure 1. Background-corrected DART-TOF mass spectra of healthy human serum in the $60-800 \mathrm{~m} / \mathrm{z}$ range: (a) nonderivatized serum sample, (b) derivatized serum sample, (c) derivatized standards mixture (identity of ions follow number between parenthesis on second column from right in Table S-1), and (d) derivatized serum sample spiked with standards. Tentative identities of metabolites observed in (b) are given in Table S-1. 
of standards and reanalyzing the serum sample using identical methods (Figure 1d). The standards spiked are listed with numbers between parentheses on the second column from right in Table S-1 and labeled in Figure 1d. Upon spiking, the intensity of the corresponding signals in Figure $1 \mathrm{~b}$ increased, thus confirming the ability of this approach to detect and identify metabolites in human serum. For redundancy and further validation, the identity of the ionic species observed for the previous standards in the absence of biological matrix was also checked by spiking a solvent and derivatization reactant mixture blank (Figure 1c). Ion adducts such as $[\mathrm{M}+\mathrm{H}]^{+},[\mathrm{M}+\mathrm{TMS}+\mathrm{H}]^{+},[\mathrm{M}+2 \mathrm{TMS}+\mathrm{H}]^{+}$, and $[\mathrm{M}+3 \mathrm{TMS}+\mathrm{H}]^{+}$were observed. These are noted with asterisks in Table S-1. These experiments reinforced the idea that analysis of TMS derivatized metabolites is preferable to the direct analysis of their more hydrophilic underivatized counterparts, despite the need for additional sample preparation steps. Intermolecular hydrogen bonding interactions in underivatized metabolite mixtures are strong, and result in decreased volatility. Derivatization replaces reactive hydrogen atoms in acidic and basic groups by TMS, leading to a reduction in mixture polarity.

\section{Effect of Helium Gas Flow Rate and Temperature}

Helium gas temperature and flow rate are two major parameters affecting DART ion transmission [24]. DART-TOF spectra for various helium gas tempera- tures and the corresponding number of metabolites identified by accurate mass matching are shown in Figure $2 a$ and $b$, respectively. As temperature was increased, the number of metabolites found was also observed to increase up to $200{ }^{\circ} \mathrm{C}$. It is important to note that temperature values refer to set values in the software, but that the local temperature where the sample is exposed to the ionizing gas stream has been measured and calculated to be lower [24]. To verify the effect of temperature, three randomly chosen signals with different $m / z$ values spanning the observed mass range were selected. A plot of their $\mathrm{S} / \mathrm{N}$ versus temperature is shown in Figure 2c, showing that the optimum temperature falls in the range of $150-200{ }^{\circ} \mathrm{C}$ depending on the $m / z$ of these metabolites. High gas temperatures accelerate sample drying and analyte thermal desorption rates, thus increasing the sensitivity of detection, but too high temperature $\left(>250^{\circ} \mathrm{C}\right)$ causes metabolites to desorb too quickly, resulting in signal loss if the spectral acquisition rate is not high enough, as would be the case with a scanning mass analyzer. High gas temperatures also lead to partial sample charring on the glass capillary surface, leading to irreversible sample degradation.

Helium flow rates were also observed to have a strong influence on the observed DART-TOF spectra (Figure 3). The number of metabolites detected increased with increased flow rate, but high gas flow (>3 L $\min ^{-1}$ ) dispersed sample particles and remaining solvent directly onto the mass spectrometer inlet, thus contaminating the inlet orifice. Moreover, high flow gas
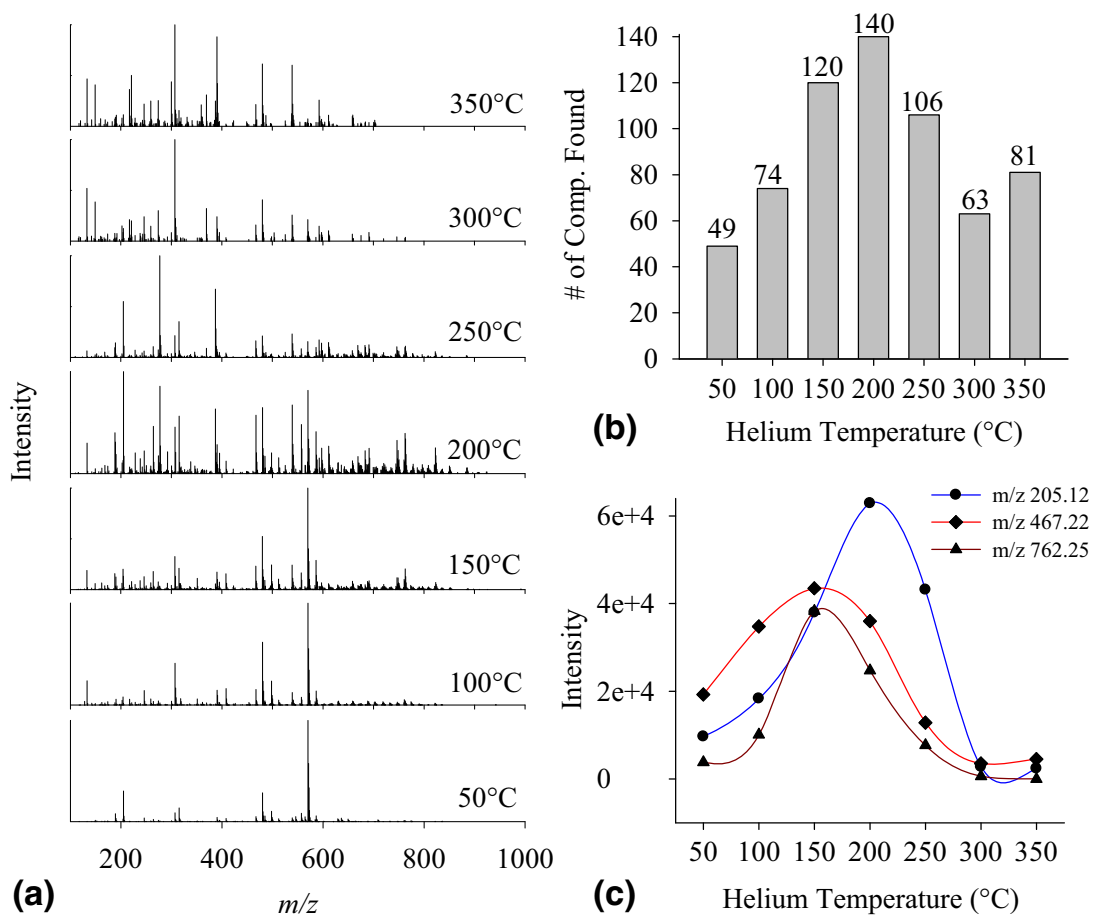

Figure 2. Effect of helium gas temperature on DART-TOF MS sensitivity for metabolomic profiling of derivatized serum: (a) background corrected mass spectra at various helium temperatures, (b) number of metabolites matched to HMDB database, and (c) change in $\mathrm{S} / \mathrm{N}$ of three mass spectrometric signals at $m / z 205.12,467.22$, and 762.25 versus helium temperature. 

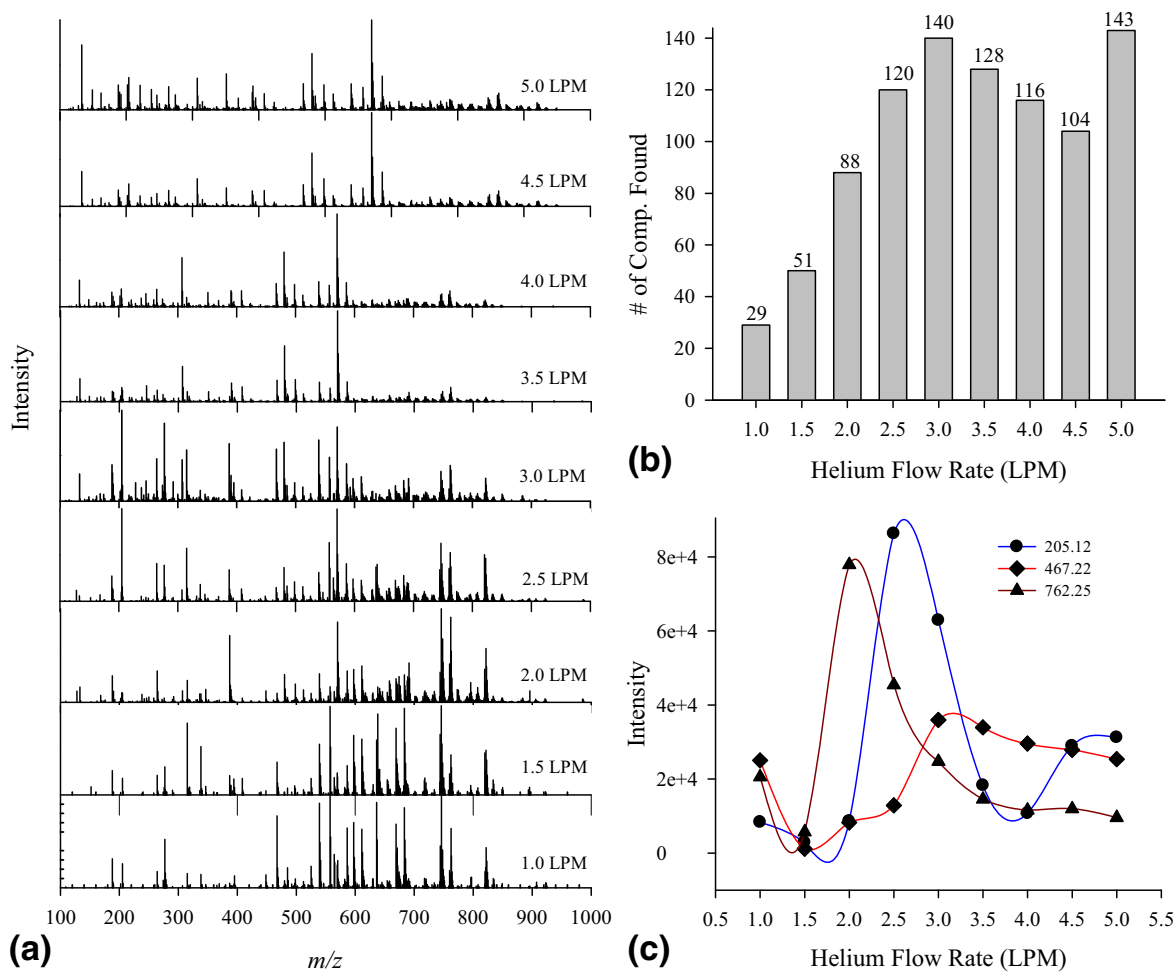

Figure 3. Helium gas flow rate effect on DART-TOF MS sensitivity for metabolomic analysis of derivatized serum. (a) Mass spectra obtained at various helium flow rates, (b) number of metabolites found by matching to HMDB database at different helium flow rates, and (c) observed S/N of mass spectrometric signals at $\mathrm{m} / \mathrm{z} 205.12,467.22$, and 762.25 .

is conducive to strong turbulence [24] and was observed to affect the reproducibility of the experiments. The S/N plots for the ionic signals previously studied indicated an optimum helium flow rate between 2.5 and 3.0 $\mathrm{L} \mathrm{min}^{-1}$ (Figure 3c).

\section{Time-Dependence of Metabolite Desorption/Ionization}

Although the underlying mechanisms prevailing in the DART desorption process are complex and beyond the scope of this study, the observed temporal profiles following exposure of the derivatized serum sample to the ionizing gas stream suggest a differential thermal desorption mechanism for most species observed during the first $5 \mathrm{~s}$ following switching of the position of the sampling arm. DART-TOF mass spectra averaged every $1 \mathrm{~s}$ of the total ion chronogram (TIC, solid curve in Figure $4 a$ ) are shown in Figure $4 b(a-h)$. At early times [Figure $4 \mathrm{~b}(a)$ ], only a few intense signals were detected, corresponding mostly to light ions. Examination of the mass spectra obtained between 40 and $44 \mathrm{~s}$ [Figure $4 \mathrm{~b}(b-e)]$, showed that these low mass signals decreased in intensity with increasing time until they completely disappeared after $42 \mathrm{~s}$ [Figure $4 \mathrm{~b}(d)$ ]. For spectra collected between 42 and $44 \mathrm{~s}$, a large quantity of signals with medium intensities at masses between $\mathrm{m} / \mathrm{z} 150$ and 800 were observed, followed by an overall decay in signal intensity at the trailing edge of the transient TIC signal. Following these experiments, we determined an optimum time interval for spectrum averaging that spans regions " $c$ " through " $e$ " in the TIC. However, it must be noted that this interval will vary depending on the type of sample holder used, mass range of the metabolites of interest, and helium flow rate and temperature.

\section{Repeatability}

Highly repeatable measurements are critical in serum metabolomic fingerprinting since potential biomarkers of stress or disease are down-selected based on significance tests or multivariate analysis of intensity information directly obtained from mass spectra. Repeatability experiments based on 10 separate runs of a control serum sample are presented in Figure $4 \mathrm{c}$ and $\mathrm{d}$. A coefficient of variation (CV) of $4.5 \%$ was obtained for the TIC peak heights shown in Figure 4c. Relative signal intensities showed good reproducibility across all spectra (Figure $4 \mathrm{~d}$ ), with an average CV of $18.9 \%$ and $16.7 \%$ for the two peaks marked with asterisks, respectively.

\section{Comparison of Metabolic Fingerprints Obtained by DART-TOF and DART-Q-TOF MS}

Experiments similar to those described above for DART-TOF MS were repeated with a Q-TOF MS instrument fitted with a DART ion source. In this instrument, 

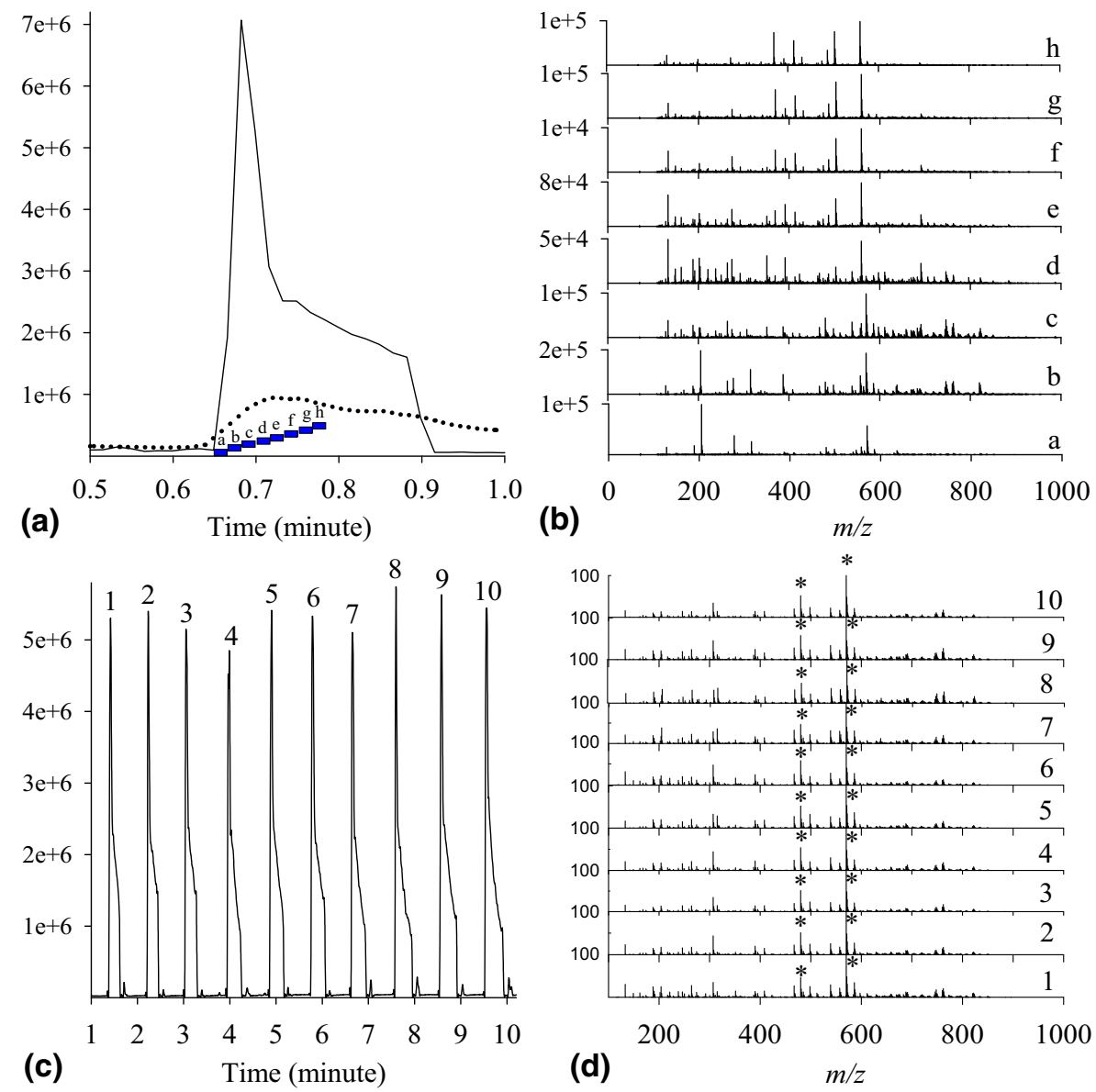

Figure 4. (a) Total ion chronogram (TIC) observed for derivatized serum (solid curve: TOF, dotted curve: Q-TOF). Each time segment denotes a time interval of $1 \mathrm{~s}$. (b) Background corrected, averaged mass spectra corresponding to each time interval. (c) Repeatability experiments for 10 manual injections of a healthy serum sample analyzed by DART-TOF MS. (d) Mass spectra corresponding to TIC peaks shown in (c). Asterisks denote signals selected for CV calculation.

the transition from atmospheric pressure to the first differentially pumped region is normally achieved with a heated capillary interface, rather than a small-diameter orifice as with the TOF mass spectrometer previously used. For efficient ion transmission into this region, a gas-ion separator tube (GIST) interface stage was added between the DART ion source outlet and the mass spectrometer. The additional pumping incorporated by this interface also serves the purpose of preventing overload of the mass spectrometer's vacuum system. Optimization experiments similar to those described above for the DART-TOF MS platform indicated an optimum temperature of $200{ }^{\circ} \mathrm{C}$ and an optimum flow rate of $0.55 \mathrm{~L} \mathrm{~min}^{-1}$. This flow rate was the minimum compatible with maintaining the TOF mass analyzer within the typical operational values recommended by the manufacturer $\left(1 \times 10^{-7}\right.$ Torr $)$. Compared with the TOF TICs (Figure $4 a$, solid curve), TICs observed for DART-Q-TOF MS were much broader (Figure $4 \mathrm{a}$, dotted curve) due to the lower flow rate of helium gas used $\left(0.55 \mathrm{~L} \mathrm{~min}^{-1}\right)$ and dispersion effects caused by the additional VAPUR interface chamber. Compared in terms of absolute signal, the Q-TOF TIC for derivatized serum was less intense, possibly because of the much longer distance between the sample and the MS inlet ( $3 \mathrm{~mm}$ for TOF, $55 \mathrm{~mm}$ for Q-TOF). However, the results for underivatized serum showed that signalto-noise ratios for Q-TOF were much better (Figure 5a) than for TOF, and that the mass range detected reached $\mathrm{m} / \mathrm{z} 500$ even for underivatized serum samples. Table S-2 lists the tentative identities of peaks observed in derivatized serum (Figure $5 b$ ) with relative intensity greater than $5 \%$ of the base peak. A total of 44 metabolites were identified, 25 of them with mass accuracy better than $1.5 \mathrm{ppm}$. Only six metabolites were detected with mass accuracy in this range by TOF MS. DARTTOF and Q-TOF mass spectra of derivatized serum were quite different in overall appearance, equivalent to different "metabolic fingerprints". Clearly, these fingerprints should not be compared across different instrumental platforms, and multivariate modeling should be based only on mass spectra obtained under identical conditions using a single instrument. The repeatability observed for derivatized human serum analysis by DART-Q-TOF MS was slightly better than for TOF-MS experiments with a CV of $4.1 \%$ for the TIC peak heights, 

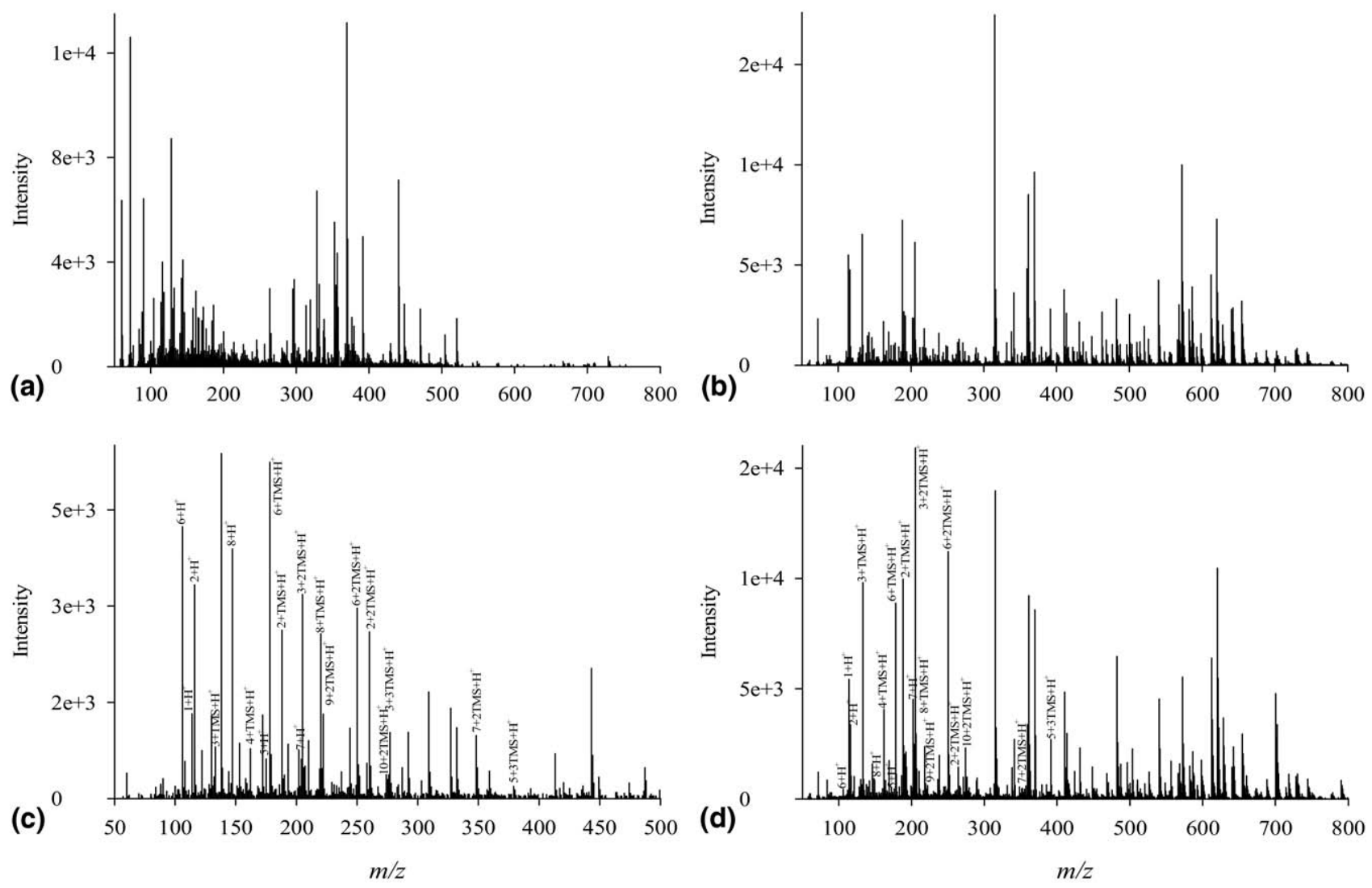

Figure 5. DART-Q-TOF mass spectra of healthy human serum in the 60-800 $\mathrm{m} / \mathrm{z}$ range: (a) nonderivatized serum sample, (b) derivatized serum sample, (c) derivatized standards mixture (identity of ions follows number between parenthesis on second column from right in Table S-2), and (d) derivatized serum sample spiked with standards. Tentative identities of metabolites observed in (b) are given in Table S-2.

and an average $\mathrm{CV}$ of $13.9 \%$ for $\mathrm{m} / \mathrm{z} 315.10$ (Supplementary Information Figure S-1,). This improved repeatability is possibly due to the use of the VAPUR ${ }^{2}$ interface which mitigates uncontrolled turbulence in the ionization region.

\section{Conclusions}

The rapid DART MS method described in this study has shown to be an efficient and sensitive approach to detect metabolites in serum. Its main attractive features include its high sample throughput, lack of crosscontamination between runs, and simplicity. The coupling of DART to accurate mass TOF mass spectrometers enables rapid monitoring of the ionic signals and tentative identification of metabolites. Further developments in automated DART spectral mining enabling batch metabolite database queries based on accurate mass, isotopic profile abundances, and accurate mass MS/MS data should further benefit this approach. Application of this method to probing changes in the human metabolome induced by disease, stress, or other abnormal conditions will provide a better perspective on the amount of biological information that may be derived from DART MS metabolomic profiles.

\section{Acknowledgments}

The authors acknowledge support from a Blanchard Assistant Professorship from the School of Chemistry and Biochemistry of the Georgia Institute of Technology to F.M.F., the Ovarian Cancer Institute at the School of Biology of the Georgia Institute of Technology to J.M., and the VentureLab Program sponsored by the Georgia Research Alliance to F.M.F. and J.M. The authors also thank Mr. Glenn Harris for insightful comments on the manuscript.

\section{Appendix A Supplementary Material}

Supplementary material associated with this article may be found in the online version at doi:10.1016/ j.jasms.2009.09.004.

\section{References}

1. Dettmer, K.; Aronov, P. A.; Hammock, B. D. Mass Spectrometry-Based Metabolomics. Mass Spectrom. Rev. 2007, 26, 51-78.

2. Ala-Korpela, M. Critical Evaluation of $1 \mathrm{H}$ NMR Metabonomics of Serum as a Methodology for Disease Risk Assessment and Diagnostics. Clin. Chem. Lab. Med. 2008, 46, 27-42.

3. Chan, E. C. Y.; Koh, P. K.; Mal, M.; Cheah, P. Y.; Eu, K. W.; Backshall, A.; Cavill, R.; Nicholson, J. K.; Keun, H. C. Metabolic Profiling of Human Colorectal Cancer Using High-Resolution Magic Angle Spinning Nuclear Magnetic Resonance (HR-MAS NMR) Spectroscopy and 
Gas Chromatography Mass Spectrometry (GC/MS). J. Proteome Res. 2009, 8, 352-361.

4. Mäkinen, V.P.; Soininen, P.; Forsblom, C.; Parkkonen, M.; Ingman, P.; Kaski, K.; Groop, P.H.; Ala-Korpela, M.; FinnDiane Study Group. Diagnosing Diabetic Nephropathy by ${ }^{1} \mathrm{H}$ NMR metabonomics of serum. MAGMA 2006, 19, 281-296.

5. Denkert, C.; Budczies, J.; Kind, T.; Weichert, W.; Tablack, P.; Sehouli, J.; Niesporek, S.; Könsgen, D.; Dietel, M.; Fiehn, O. Mass SpectrometryBased Metabolic Profiling Reveals Different Metabolite Patterns in Invasive Ovarian Carcinomas and Ovarian Borderline Tumors. Cancer Res. 2006, 66, 10795-10804.

6. Fancy, S.A.; Beckonert, O.; Darbon, G.; Yabsley, W.; Walley, R.; Baker, D.; Perkins, G. L.; Pullen, F. S.; Rumpel, K. Gas Chromatography/Flame Ionization Detection Mass Spectrometry for the Detection of Endogenous Urine Metabolites for Metabonomic Studies and Its Use as a Complementary Tool to Nuclear Magnetic Resonance Spectroscopy. Rapid Commun. Mass Spectrom. 2006, 20, 2271-2280.

7. Lu, W.; Bennett, B. D.; Rabinowitz, J. D. Analytical Strategies for LC-MS-Based Targeted Metabolomics. J. Chromatogr. 2008, 871, 236-242.

8. Michopoulos, F.; Lai, L.; Gika, H.; Theodoridis, G.; Wilson, I. UPLCMS-Based Analysis of Human Plasma for Metabonomics Using Solvent Precipitation or Solid Phase Extraction. J. Proteome Res. 2009, 8, 2114 2121.

9. Pasikanti, K. K.; Ho, P. C.; Chan, E. C. Y. Gas Chromatography/Mass Spectrometry in Metabolic Profiling of Biological Fluids. J. Chromatogr. 2008, 871, 202-211.

10. Haapala, M.; Pol, J.; Saarela, V.; Arvola, V.; Kotiaho, T.; Ketola, R. A.; Franssila, S.; Kauppila, T. J.; Kostiainen, R. Desorption Atmospheric Pressure Photoionization. Anal. Chem. 2007, 79, 7867-7872.

11. Cody, R. B.; Larame, J. A.; Durst, H. D. Versatile New Ion Source for the Analysis of Materials in Open Air Under Ambient Conditions. Anal. Chem. 2005, 77, 2297-2302.

12. Andrade, F. J.; Shelley, J. T.; Wetzel, W. C.; Webb, M. R.; Gamez, G.; Ray, S. J.; Hieftje, G. M. Atmospheric Pressure Chemical Ionization Source. 2. Desorption-Ionization for the Direct Analysis of Solid Compounds. Anal. Chem. 2008, 80, 2654-2663.

13. Ratcliffe, L. V.; Rutten, F. J.; Barrett, D. A.; Whitmore, T.; Seymour, D.; Greenwood, C.; Aranda-Gonzalvo, Y.; Robinson, S.; McCoustra, M. Surface Analysis Under Ambient Conditions Using Plasma-Assisted Desorption/Ionization Mass Spectrometry. Anal. Chem. 2007, 79, 60946101.
14. Jackson, A. T.; Scrivens, J. H.; Williams, J. P.; Baker, E. S.; Gidden, J.; Bowers, M. T. Microstructural and Conformational Studies of Polyether Copolymers. Int. J. Mass Spectrom. 2004, 238, 287-297.

15. Harper, J. D.; Charipar, N. A.; Mulligan, C. C.; Zhang, X.; Cooks, R. G.; Ouyang, Z. Low-Temperature Plasma Probe for Ambient Desorption Ionization. Anal. Chem. 2008, 80, 9097-9104.

16. Na, N.; Zhang, C.; Zhao, M.; Zhang, S.; Yang, C.; Fang, X.; Zhang, X. Direct Detection of Explosives on Solid Surfaces by Mass Spectrometry with an Ambient Ion Source Based on Dielectric Barrier Discharge. J. Mass Spectrom. 2007, 42, 1079-1085.

17. Petucci, C.; Diffendal, J.; Kaufman, D.; Mekonnen, B.; Terefenko, G. Musselman, B. Direct Analysis in Real Time for Reaction Monitoring in Drug Discovery. Anal. Chem. 2007, 79, 5064-5070.

18. Zhao, Y.; Lam, M.; Wu, D.; Mak, R. Quantification of Small Molecules in Plasma with Direct Analysis in Real Time Tandem Mass Spectrometry, Without Sample Preparation and Liquid Chromatographic Separation. Rapid Commun. Mass Spectrom. 2008, 22, 3217-3224.

19. Fernandez, F. M.; Cody, R. B.; Green, M. D.; Hampton, C. Y.; McGready R.; Sengaloundeth, S.; White, N. J.; Newton, P. N. Characterization of Solid Counterfeit Drug Samples by Desorption Electrospray Ionization and Direct Analysis-in-Real-Time Coupled to Time-of-Flight Mass Spectrometry. Chem. Med. Chem. 2006, 1, 702-705.

20. Pierce, C. Y.; Barr, J. R.; Cody, R. B.; Massung, R. F.; Woolfitt, A. R. Moura, H.; Thompson, H. A.; Fernandez, F. M. Ambient Generation of Fatty Acid Methyl Ester Ions from Bacterial Whole Cells by Direct Analysis in Real Time (DART) Mass Spectrometry. Chem. Commun. 2007, 8, 807-809.

21. Haefliger, O. P.; Jeckelmann, N. Direct Mass Spectrometric Analysis of Flavors and Fragrances in Real Applications Using DART. Rapid Commun. Mass Spectrom. 2007, 21, 1361-1366.

22. Schurek, J.; Vaclavik, L.; Hooijerink, H. D.; Lacina, O.; Poustka, J. Sharman, M.; Caldow, M.; Nielen, M. W. F.; Hajslova, J. Control of Strobilurin Fungicides in Wheat Using Direct Analysis in Real Time Accurate Time-of-Flight and Desorption Electrospray Ionization Linear Ion Trap Mass Spectrometry. Anal. Chem. 2008, 80, 9567-9575.

23. Williams, J. P.; Patel, V. J.; Holland, R.; Scrivens, J. H. The Use of Recently Described Ionization Techniques for the Rapid Analysis of Some Common Drugs and Samples of Biological Origin. Rapid Commun. Mass Spectrom. 2006, 20, 1447-1456.

24. Harris, G. A.; Fernandez, F. M. Simulations and Experimental Investigation of Atmospheric Transport in an Ambient Metastable-Induced Chemical Ionization Source. Anal. Chem. 2009, 81, 322-329. 